Supporting Information for

\title{
Understanding the Polypharmacological Profiles of Triple Reuptake Inhibitors by Molecular Simulation
}

\author{
Gao Tu${ }^{1}$, Tingting Fu ${ }^{1,2}$, Fengyuan Yang ${ }^{1,2}$, Jingyi Yang ${ }^{1}$, Zhao Zhang ${ }^{1}$, Xiaojun Yao $^{3}$, Weiwei \\ $\mathrm{Xue}^{1,4 *}$, Feng $\mathrm{Zhu}^{1,2 *}$
}

${ }^{1}$ School of Pharmaceutical Sciences, Chongqing Key Laboratory of Natural Product Synthesis and Drug Research, Chongqing University, Chongqing 401331, China

${ }^{2}$ College of Pharmaceutical Sciences, Zhejiang University, Hangzhou 310058, China

${ }^{3}$ State Key Laboratory of Applied Organic Chemistry and Department of Chemistry, Lanzhou University, Lanzhou 730000, China

${ }^{4}$ Central Nervous System Drug Key Laboratory of Sichuan Province, Luzhou 646106, China

\author{
*Corresponding Author \\ Dr. Weiwei Xue \\ E-mail:xueww@cqu.edu.cn \\ Dr. Feng Zhu \\ E-mail: zhufeng@zju.edu.cn
}




\section{SI RESULTS AND DISCUSSION}

\section{Structural Evaluation of hDAT, hNET and hSERT}

The 3D structure modeled of hDAT and hNET using the crystallographic structure of escitalopram bound to hSERT (PDB code: $5 \mathrm{I} 71^{1}$ ). The overall sequence identity between hSERT and the modeled structure (hDAT and hNET) are $52.30 \%$ and $53.60 \%$ (Figure S1), respectively. The detailed evaluation of models in Figure $\mathbf{S 2}$ to $\mathbf{S 4}$ shows the reliability of the modified hSERT, the modeled hDAT and hNET can be used for investigating the binding of NS2359, SEP225289 and EB1020 to their targets.

\section{Binding Mode of the Nine Complexes Derived from MD simulation}

\section{hDAT Bound Complexes}

The binding modes of EB1020, SEP225289 and NS2359 in hDAT were displayed in Figure 4A to 4C. As shown, the binding site is composed of residues F76, A77, D79, S149, V152, G153, Y156, F320, S321, G323, F326, V328, S422, A423, G426, M427 and I484. Hydrophobic interactions and hydrogen bond interactions are the principal driving force for the formation of the complexes. For SEP225289-bound hDAT complex, the positively charged nitrogen moiety of the SEP225289 established hydrogen bond interaction with the side chains of residues D79 and the backbone oxygen atoms of S321 and F320 in hDAT. The 3,4-dichlorophenyl of SEP225289 is mainly flanked by TM3 and TM8 (S149, V152, G153, Y156, A423, G426 and M427) constituting hydrophobic interactions within a hydrophobic binding pocket, particularly involves the strong hydrophobic interaction between aromatic residues F76, Y156, F320 and F326, as illustrated in Figure 4A. For NS2359-bound hDAT complex, the positively charged nitrogen moiety of the NS2359 engaged in hydrogen bond interaction with the side chains of residues D79 and Y156. The tropane moiety and methoxymethyl group of NS2359 kept in close proximity to TM1 (D79 and F76) and TM6 (F320, S321, G323, F326 and V328) region, which are involved in hydrophobic interactions. The 3,4-dichlorophenyl group of the NS2359 extending into the protein TM3 and TM8 regions composed of residues S149, V152, Y156, S422, A423, G426 and M427 that can interact and make hydrophobic interaction with ligand (Figure 4B). For EB1020-bound hDAT complex, the charged residue D79 engaged in hydrogen bond with the charged nitrogen moiety of the EB1020. Meanwhile, the protonated nitrogen of the EB1020 interacts simultaneously with the carbonyl group and side chains of residue F76 formed hydrogen bond interaction and cation- $\pi$ interaction, respectively(Figure 4C). The naphthyl group of EB1020 makes interactions with the mainly hydrophobic residues (V152, Y156, A423 and M427) of the TM3 
and TM8 in hDAT.

\section{hNET Bound Complexes}

Figure 4D to 4F shown that the binding pocket of hNET was formed by residues F72, A73, D75, A145, V148, Y152, F317, S318, L319, G320, F323, V325, S419, S420, G423, M424 and V449. Hydrophobic interactions and hydrogen bond interactions are primarily responsible for the binding of hNET bound complexes. For SEP225289-bound hNET complex, the protonated nitrogen of SEP225289 engaged in two hydrogen bonds interaction with charged residue D75 and oxygen of F317.The hydrophobic site in the TM3 and TM8 domains (A145, V148, G149, Y152, S419, S420, G423 and M424) established hydrophobic interactions with the 3,4-dichlorophenyl group of SEP225289. In addition, the side chains of residue Y152 participate in face-to-face $\pi$-stacking interaction with SEP225289 (Figure 4D). For NS2359-bound hNET complex system, the tropane moiety of the ligand occupied the regions of TM1 and TM6 and the protonated nitrogen of NS2359 engaged in three hydrogen bonds interaction with charged residue D75, the side chains of residue Y152 and backbone carbonyl oxygen of residue S318, respectively. (Figure 4E). The 3,4dichlorophenyl group of ligand close to the residues (V148, G149, S420, G423, S419 and Y152) and formed hydrophobic interactions in hNET. For EB1020-bound hNET complex, the positively charged nitrogen moiety of the EB120 established three hydrogen bonds interactions with the conserved residues D75, the backbone carbonyl oxygen of residue S318 and F72 in hNET. The naphthyl group of EB1020 make hydrophobic interactions with the surrounding residues (V148, G423, Y152, S420 and S419) of TM3 and TM8 domains (Figure 4F).

\section{hSERT Bound Complexes}

The active site of hSERT consists of residues (Y95, A96, D98, A169, I172, A173, Y176, F335, S336, L337, G338, F341, V343, S438, T439, G442 and L443) that formed hydrophobic interactions and hydrogen bond interactions with EB1020, SEP225289 and NS2359 binding site (Figure 4G to 4I). For SEP225289-bound hSERT complex system, three hydrogen bonds link the protonated nitrogen of SEP225289 to the side chains of D98 and backbone carbonyl oxygen of residue (F335 and S336) in the binding pocket. Hydrophobic interactions that formed between the 3, 4-dichlorophenyl group of SEP225289 and residues (I172, L443, A173, T431, G442, Y176 and S438) (Figure 4G). For NS2359-bound hSERT complex, the protonated nitrogen of NS2359 and residue D98, residue S438 and backbone carbonyl oxygen Y95 are primarily engaged in three hydrogen bonds interaction (Figure 4H). The 3,4-dichlorophenyl group of the NS2359 extending into the protein TM3 and TM8 regions composed of residues A169, I172, A173, L443, Y176, T431 and G442 that can interact and 
participate in hydrophobic interaction with NS2359. For EB1020-bound hSERT complex, the protonated nitrogen of EB1020 make a favorable hydrogen bond interaction with residue D98 and backbone carbonyl oxygen of residue Y95. Meanwhile, the protonated nitrogen of the EB1020 formed additional face-to-face $\pi$-stacking interaction with the side chains of residue D98. The naphthyl group of EB1020 is involved in hydrophobic interactions to the residues I172, A173, L443, Y176 and T439 in hSERT (Figure 4I).

\section{Binding Mode of Three Substrates Complex Derived from Docking Model}

For DA-hDAT complex, the positively charged nitrogen moiety of the DA established two hydrogen bonds interaction with the side chains of residues D79 and S422. Residues S149, Y156, M427, S429 and F326 form hydrophobic interactions with DA (Figure S6A). For NE-hNET complex, the protonated nitrogen of NE engaged in three hydrogen bonds interaction with charged residue A73, D75 and S419.The hydrophobic site in the hNET (F72, V148, Y152, S419, S420 and M424) established hydrophobic interactions with NE (Figure S6B). For 5-HT-hSERT complex, one hydrogen bond link the protonated nitrogen of 5-HT to the side chains of D98, hydrophobic interactions that formed between the 5-HT and residues I172, L443, A173, T431, Y176 and S438 (Figure S6C). 
Table S1. List of triple reuptake inhibitors (TRIs) entered into clinical trials ${ }^{2}$

\begin{tabular}{|c|c|c|c|c|}
\hline $\begin{array}{l}\text { Triple reuptake } \\
\text { inhibitors }\end{array}$ & Structures & $\begin{array}{l}\text { Highest } \\
\text { stage of development }\end{array}$ & Diseases & $\begin{array}{l}\text { Activities (nM) } \\
\text { DAT/NET/SERT }\end{array}$ \\
\hline $\begin{array}{l}\text { SEP225289 } \\
\text { (Dasotraline) }\end{array}$ & & Phase 3 & $\mathrm{MDD}, \mathrm{BED}$ and ADHD & $2 / 4 / 14$ \\
\hline $\begin{array}{l}\text { NS2359 } \\
(\text { GSk-372475) }\end{array}$ & & Phase 2 & $\begin{array}{l}\text { MDD, ADHD and } \\
\text { cocaine addiction }\end{array}$ & $10 / 2 / 10$ \\
\hline $\begin{array}{l}\text { Centanafadine } \\
\text { (EB1020) }\end{array}$ & & Phase 3 & MDD and ADHD & $38 / 6 / 84$ \\
\hline $\begin{array}{l}\text { Amitifadine } \\
\text { (DOV-21947) }\end{array}$ & & Phase 3 & MDD & $96 / 23 / 12$ \\
\hline RG-7166 & & Phase 1 & MDD & $90 / 9 / 16$ \\
\hline GSK1360707 & & Phase 1 & MDD & $8.0 / 8.1 / 9.2$ \\
\hline
\end{tabular}


Table S2. The per-residue energy of the residues in hDAT contribute to SEP225289, NS2359 and EB1020 binding ( $\Delta G_{\text {per-residue }}$ is in $\left.\mathrm{kcal} / \mathrm{mol}\right)$

\begin{tabular}{|c|c|c|c|}
\hline \multirow{2}{*}{ Residues } & \multicolumn{3}{|c|}{ Per-residue energy contribution ( $\left.\Delta G_{\text {per-residue }}\right)$} \\
\hline & hDAT-SEP225289 & hDAT-NS2359 & hDAT-EB1020 \\
\hline F76 & -1.9 & -2.47 & -3.81 \\
\hline A77 & -0.45 & -0.70 & -0.52 \\
\hline D79 & -3.68 & -2.99 & -3.25 \\
\hline S149 & -0.59 & -0.92 & -0.50 \\
\hline V152 & -1.71 & -1.72 & -1.34 \\
\hline G153 & -0.77 & -0.49 & -0.44 \\
\hline Y156 & -2.95 & -2.55 & -1.22 \\
\hline F320 & -1.74 & -0.65 & -0.73 \\
\hline S321 & -1.64 & -0.93 & -1.19 \\
\hline G323 & -0.38 & -1.00 & -0.73 \\
\hline F326 & -2.45 & -0.74 & -1.27 \\
\hline V328 & -0.14 & -0.78 & -0.18 \\
\hline S422 & -1.01 & -0.52 & -0.80 \\
\hline A423 & -1.04 & -1.29 & -0.65 \\
\hline G426 & -1.19 & -0.84 & -1.21 \\
\hline M427 & -0.92 & -1.00 & -0.61 \\
\hline
\end{tabular}


Table S3. The per-residue energy of the residues in hNET contribute to SEP225289, NS2359 and EB1020 binding $\left(\Delta G_{\text {per-residue }}\right.$ is in $\left.\mathrm{kcal} / \mathrm{mol}\right)$

\begin{tabular}{|c|c|c|c|}
\hline \multirow{2}{*}{ Residues } & \multicolumn{3}{|c|}{ Per-residue energy contribution ( $\left.\Delta G_{\text {per-residue }}\right)$} \\
\hline & hNET-SEP225289 & hNET-NS2359 & hNET-EB1020 \\
\hline F72 & -1.17 & -2.65 & -4.10 \\
\hline A73 & -0.23 & -1.00 & -1.21 \\
\hline D75 & -2.58 & -3.08 & -3.39 \\
\hline A145 & -0.70 & -0.51 & -0.46 \\
\hline V148 & -1.55 & -2.24 & -1.60 \\
\hline G149 & -1.53 & -0.60 & -0.43 \\
\hline Y152 & -3.16 & -2.87 & -1.97 \\
\hline F317 & -2.08 & -0.82 & -0.05 \\
\hline S318 & -0.54 & -1.15 & -1.40 \\
\hline G320 & -0.49 & -0.62 & -0.81 \\
\hline F323 & -0.13 & -1.52 & -1.21 \\
\hline V325 & -0.40 & -0.41 & -1.01 \\
\hline S419 & -0.84 & -1.12 & -0.50 \\
\hline S420 & -1.83 & -0.84 & -0.64 \\
\hline G423 & -1.11 & -0.71 & -0.77 \\
\hline M424 & -0.97 & -0.52 & -0.29 \\
\hline
\end{tabular}


Table S4. The per-residue energy of the residues in hSERT contribute to SEP225289, NS2359 and EB1020 binding $\left(\Delta G_{\text {per-residue }}\right.$ is in $\left.\mathrm{kcal} / \mathrm{mol}\right)$

\begin{tabular}{llll}
\hline \multirow{2}{*}{ Residues } & \multicolumn{2}{l}{ Per-residue energy contribution $\left(\Delta G_{\text {per-residue }}\right)$} & \\
\cline { 2 - 4 } & hSERT-SEP225289 & hSERT-NS2359 & hSERT-EB1020 \\
\hline Y95 & -2.14 & -2.82 & -2.95 \\
A96 & -0.71 & -0.50 & -0.41 \\
D98 & -2.89 & -1.12 & -1.20 \\
A169 & -0.47 & -0.63 & -0.71 \\
I172 & -2.47 & -2.29 & -1.84 \\
A173 & -0.51 & -0.69 & -0.57 \\
Y176 & -2.12 & -1.21 & -1.90 \\
F335 & -1.75 & -0.42 & -0.64 \\
S336 & -1.19 & -0.39 & -0.45 \\
G338 & -0.99 & -0.68 & -0.44 \\
F341 & -0.50 & -0.96 & -1.47 \\
V343 & -0.30 & -0.55 & -0.23 \\
S438 & -1.30 & -1.27 & -0.63 \\
T439 & -1.40 & -1.14 & -1.18 \\
G442 & -0.84 & -1.38 & -1.33 \\
L443 & -0.71 & -1.05 & -98 \\
\hline & & & \\
\hline
\end{tabular}


Table S5. Fingerprints of molecular interaction including the interaction types between hDAT and three studied TRIs

\begin{tabular}{lllll}
\hline Residues & Interaction type & EB1020 & NS2359 & SEP225289 \\
\hline F76 & $\begin{array}{l}\text { Hydrophobic } \\
\text { +H-bond (ligand donor) }\end{array}$ & 1000100 & & \\
D79 & $\begin{array}{l}\text { Hydrophobic } \\
\text { +H-bond (ligand donor) }\end{array}$ & 1000100 & 1000100 & 1000100 \\
I148 & & 000000 & 0000000 & 0000000 \\
S149 & Hydrophobic & 1000000 & & \\
V152 & Hydrophobic & 1000000 & & 1000000 \\
Y156 & Hydrophobic & 1000000 & 1000100 & 1000100 \\
F320 & Hydrophobic & 1000000 & 0000000 & 1000000 \\
F326 & Hydrophobic & 1000000 & 0000000 & 0000000 \\
V328 & Hydrophobic & 0000000 & 1000000 & \\
S422 & Hydrophobic & 1000000 & & 0000000 \\
A423 & Hydrophobic & 1000000 & 1000000 & \\
M427 & Hydrophobic & 1000000 & & \\
V430 & Hydrophobic & 1000000 & & \\
A480 & Hydrophobic & 0000000 & 0000000 & \\
I484 & Hydrophobic & 0000000 & & \\
\hline & & & \\
\hline
\end{tabular}


Table S6. Fingerprints of molecular interaction including the interaction types between hNET and three studied TRIs

\begin{tabular}{|c|c|c|c|c|}
\hline Residues & Interaction type & EB1020 & NS2359 & SEP225289 \\
\hline F72 & $\begin{array}{l}\text { Hydrophobic } \\
+ \text { H-bond (ligand donor) }\end{array}$ & 1000100 & & \\
\hline A73 & & 0000100 & 1000000 & 0000000 \\
\hline D75 & $\begin{array}{l}\text { Hydrophobic } \\
+ \text { H-bond (ligand donor) }\end{array}$ & 1000100 & 1000100 & 0000100 \\
\hline $\mathrm{I} 144$ & & 0000000 & 1000000 & 0000000 \\
\hline A145 & Hydrophobic & 1000000 & & \\
\hline V148 & Hydrophobic & 1000000 & & \\
\hline Y152 & & 1000000 & 1000100 & 1100100 \\
\hline F316 & & 0000000 & 1000000 & 0000000 \\
\hline F317 & & 0000000 & 1000000 & 1000100 \\
\hline S318 & & 0000100 & 1000000 & 0000000 \\
\hline F323 & & 0000000 & 1000000 & 0000000 \\
\hline V325 & Hydrophobic & 1000000 & & \\
\hline S419 & & 1000000 & 1000000 & 1000100 \\
\hline S429 & Hydrophobic & 1000000 & & \\
\hline M424 & Hydrophobic & 1000000 & 1000000 & 0000000 \\
\hline $\mathrm{I} 481$ & & 0000000 & 0000000 & 1000000 \\
\hline
\end{tabular}


Table S7. Fingerprints of molecular interaction including the interaction types between hSERT and three studied TRIs

\begin{tabular}{lllll}
\hline Residues & Interaction type & EB1020 & SEP225289 & NS2359 \\
\hline Y95 & Hydrophobic & 10000000 & & \\
A96 & & 0000100 & 0000000 & 0000000 \\
D98 & $\begin{array}{l}\text { Hydrophobic } \\
\text { +H-bond (ligand donor) }\end{array}$ & 1000100 & 0000100 & 1000100 \\
A169 & Hydrophobic & 1000000 & & \\
I172 & Hydrophobic & 1000000 & & \\
A173 & Hydrophobic & 1000000 & & 1000100 \\
Y176 & & 1000000 & 10000000 & 10000000 \\
F335 & & 1000100 & 0000100 & 0000000 \\
S336 & H-bond (ligand donor) & 0000100 & 0000100 & 0000000 \\
L337 & & 0000000 & 0000100 & \\
F341 & Hydrophobic & 10000000 & & 1000100 \\
V343 & Hydrophobic & 10000000 & & \\
S438 & & 0000000 & 10000000 & \\
T439 & Hydrophobic & 10000000 & 10000000 & \\
L443 & Hydrophobic & 10000000 & & \\
V446 & Hydrophobic & 10000000 & & \\
V501 & Hydrophobic & 0000000 & \\
\hline
\end{tabular}


Table S8. Summary of nine complexes for MD simulations.

\begin{tabular}{lllllll}
\hline Complexes & $\mathrm{Na}^{+}$ions & $\mathrm{Cl}^{-}$ions & POPC & water & Total atoms & Simulation time \\
\hline hDAT-SEP225289 & 61 & 53 & 219 & 19535 & 96644 & $100 \mathrm{~ns}$ \\
hDAT-NS2359 & 60 & 52 & 152 & 19123 & 86451 & $100 \mathrm{~ns}$ \\
hDAT-EB1020 & 73 & 65 & 214 & 23745 & 108625 & $100 \mathrm{~ns}$ \\
hNET-SEP225289 & 51 & 49 & 197 & 18172 & 89783 & $100 \mathrm{~ns}$ \\
hNET-NS2359 & 57 & 55 & 201 & 20539 & 97436 & $100 \mathrm{~ns}$ \\
hNET-EB1020 & 57 & 55 & 201 & 20505 & 97327 & $100 \mathrm{~ns}$ \\
hSERT-SEP225289 & 52 & 55 & 192 & 18783 & 90891 & $100 \mathrm{~ns}$ \\
hSERT-NS2359 & 52 & 55 & 191 & 18764 & 90704 & $100 \mathrm{~ns}$ \\
hSERT-EB1020 & 50 & 53 & 185 & 17854 & 87159 & $100 \mathrm{~ns}$ \\
\hline
\end{tabular}




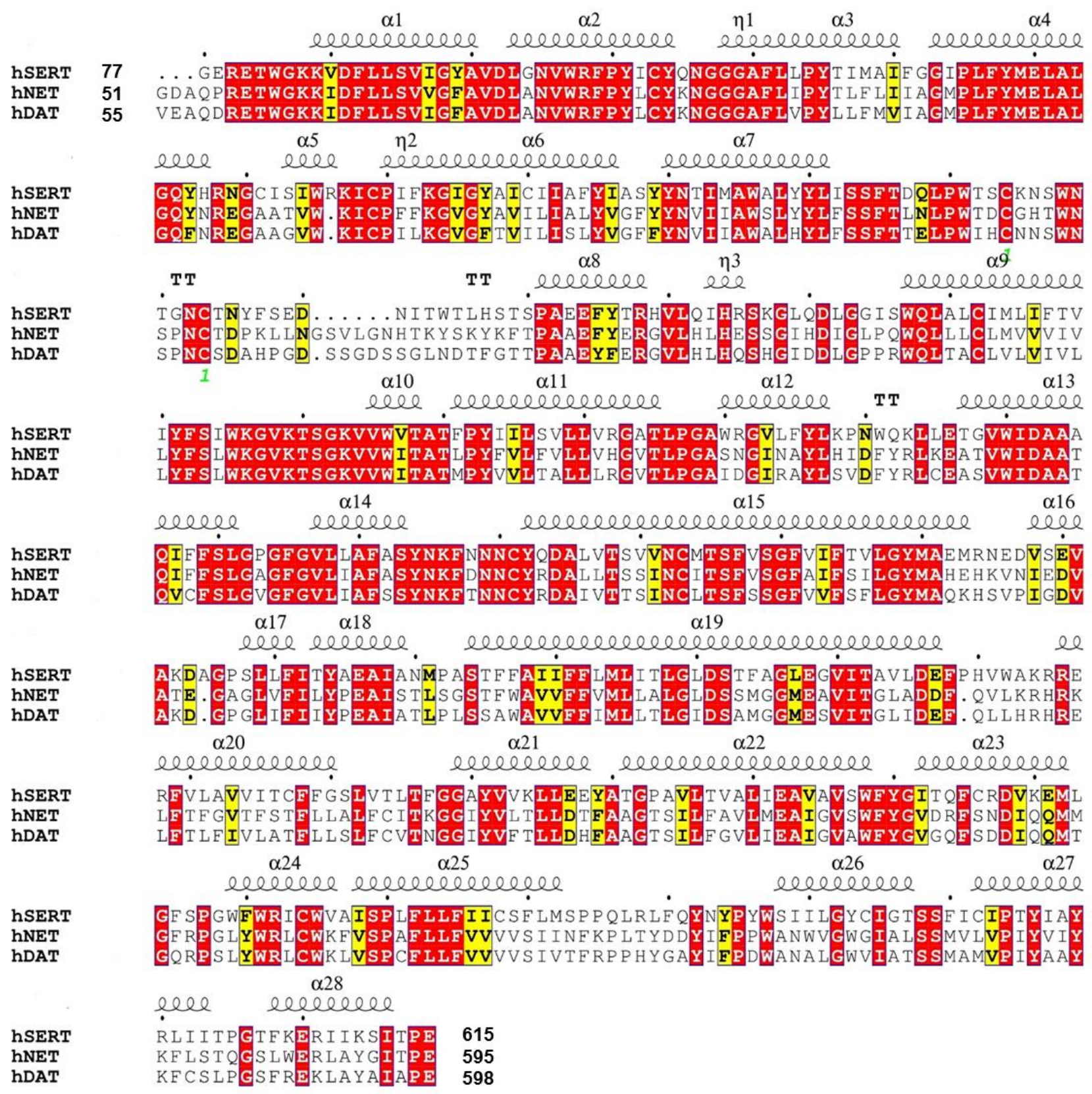

Figure S1. Sequence alignment of hSERT (from Gly77 to Glu615), hNET (from Gly51 to Glu595) and hDAT (from Val55 to Glu698) using ClustalW2 ${ }^{3}$ and visualized by ESPript $3{ }^{4}$. The sequence identities between hSERT and hNET, hDAT were 53.60\% and 52.30\%, respectively. The conserved residues are shown as white text on red background and the residue with similar properties are shown as white text on yellow background. 

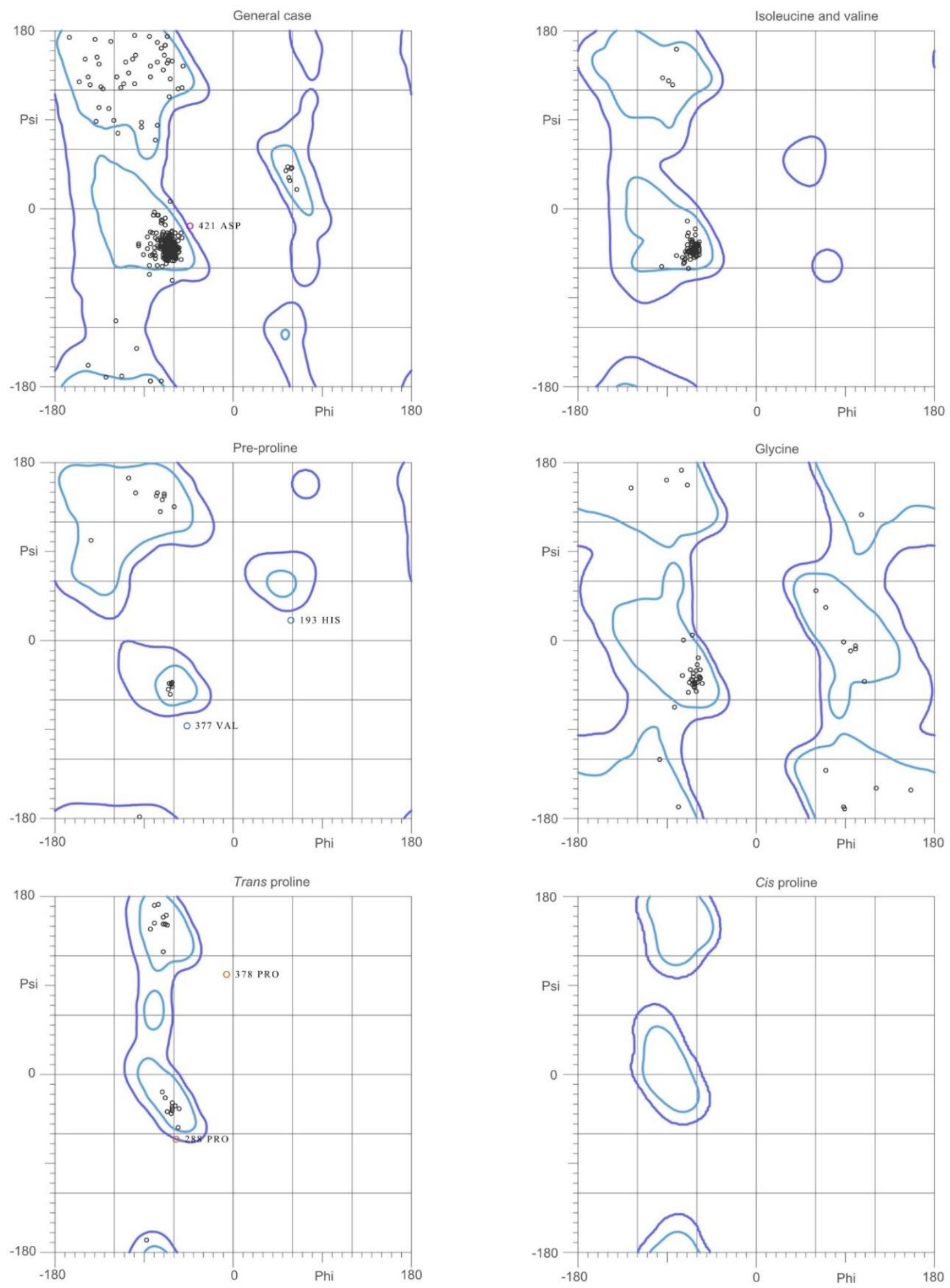

Figure S2. MolProbity Ramachandran analysis of the modeled hDAT structure. 96.7\% (523/541) of all residues were in favored (98\%) regions. 99.1\% (536/541) of all residues were in allowed (>99.8\%) regions. There were 5 outliers (phi, psi): His 193 (58.3, 21.8), Pro288 (-58.4, -65.2), Val377 (-47.7, 86.0), Pro378 (-7.8,102.0) and Asp421 (-44.8,-17.7). 

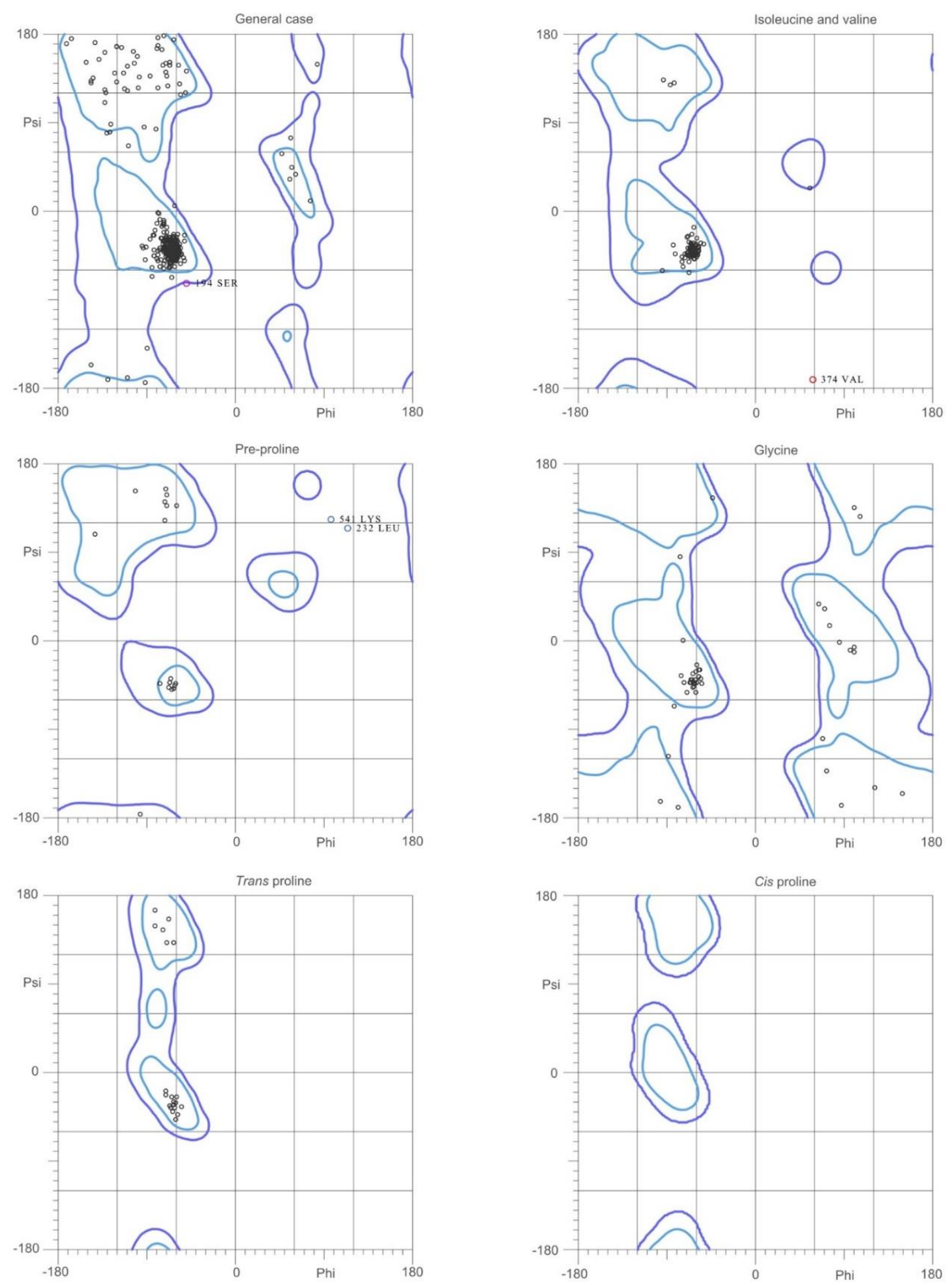

Figure S3. MolProbity Ramachandran analysis of the modeled hNET structure. 95.8\% (520/543) of all residues were in favored (98\%) regions. 99.3\% (539/543) of all residues were in allowed (>99.8\%) regions. There were 4 outliers (phi, psi): Ser194 (-50.5, -73.6), Leu232 (114.3, 115.9), Val374 (58.5, -171.3), Lys541 (97.3,124.1). 

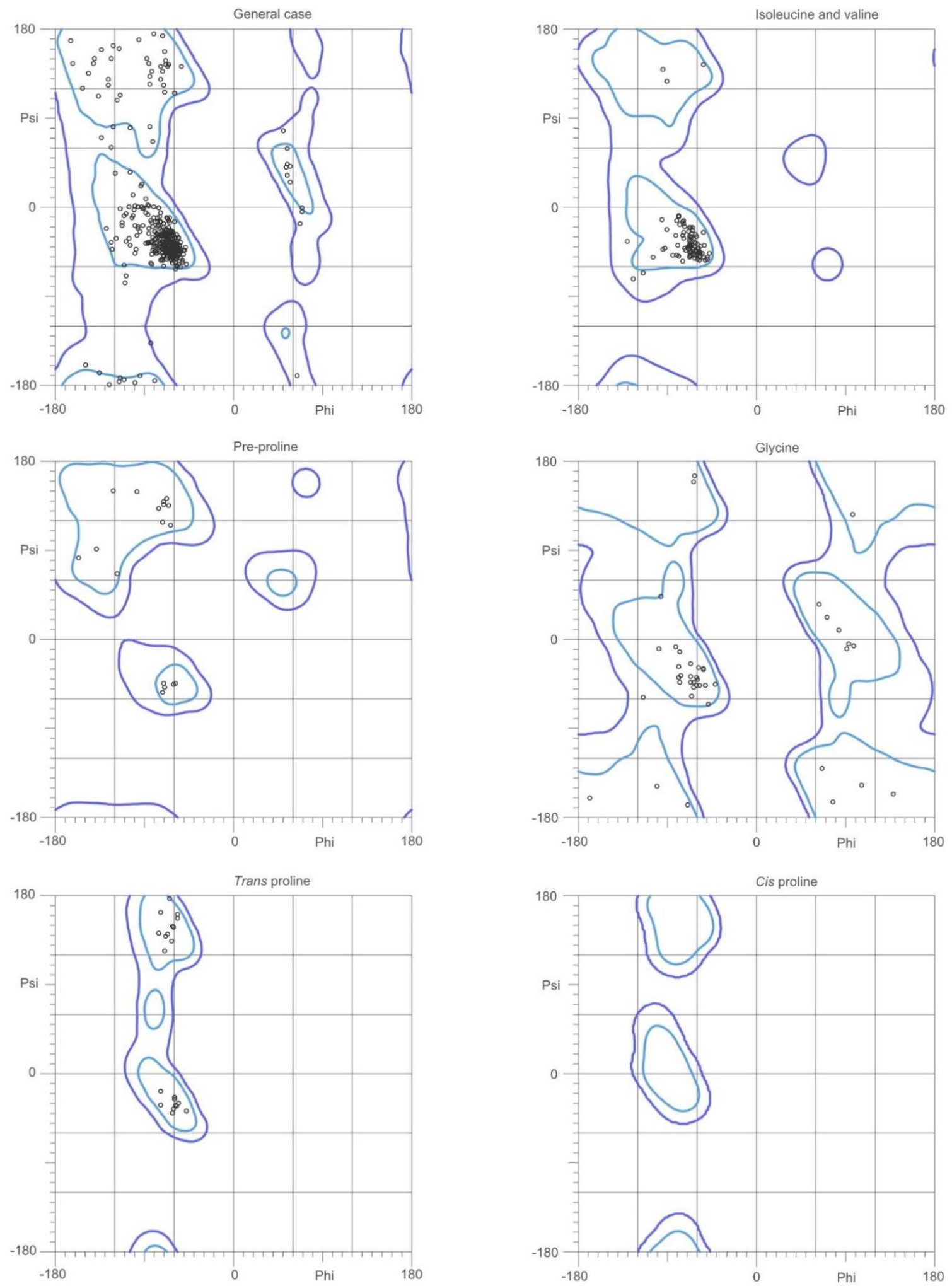

Figure S4. MolProbity Ramachandran analysis of the modeled hSERT structure. 96.8\% (518/535) of all residues were in favored (98\%) region. $100.0 \%(535 / 535)$ of all residues were in allowed $(>99.8 \%)$ regions. 

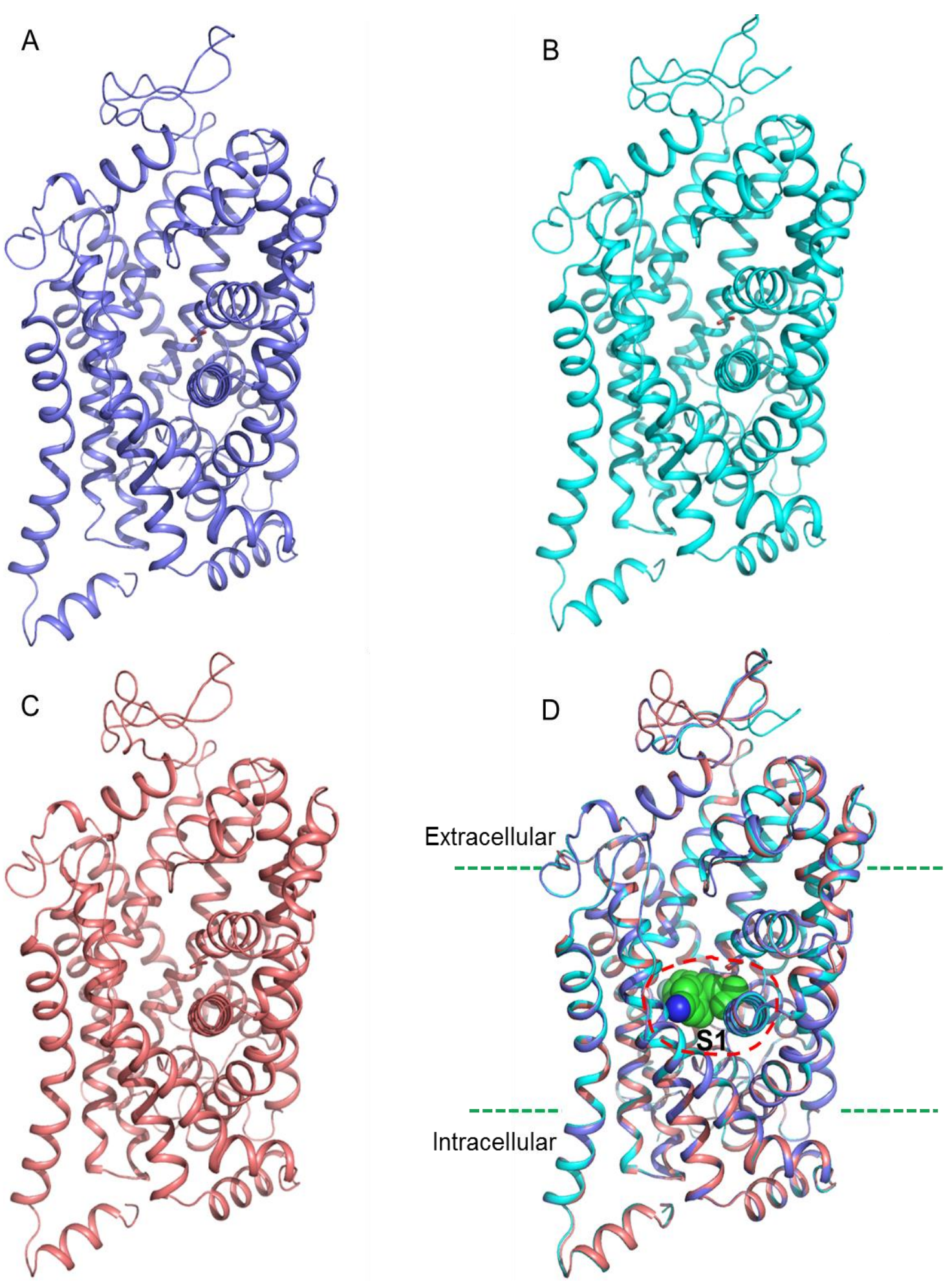

Figure S5. (A-C) Cartoon representation of the modified hSERT (blue), the modeled hNET (cyan) and hDAT (warmpink), respectively. (D) Superposition of the three hMATs with the co-crystallized escitalopram in hSERT (PDB code: $5 \mathrm{I} 71^{l}$ ) shown in green sphere and the central binding sites highlighted in red dashed lines. 


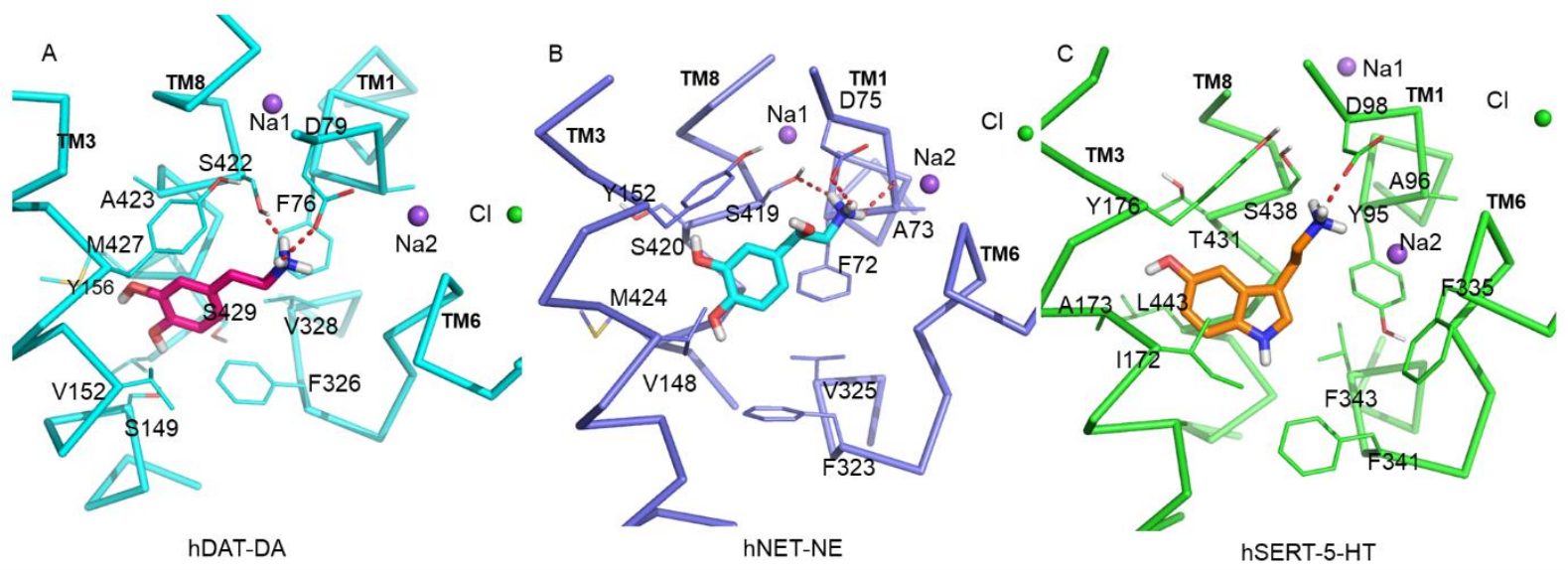

Figure S6. The docking poses of hDAT-DA (cyan), hNET-NE (blue) and hSERT-5-HT (green) complex. The substrate DA, NE and 5-HT are depicted in red, cyan, and orange stick, respectively. 

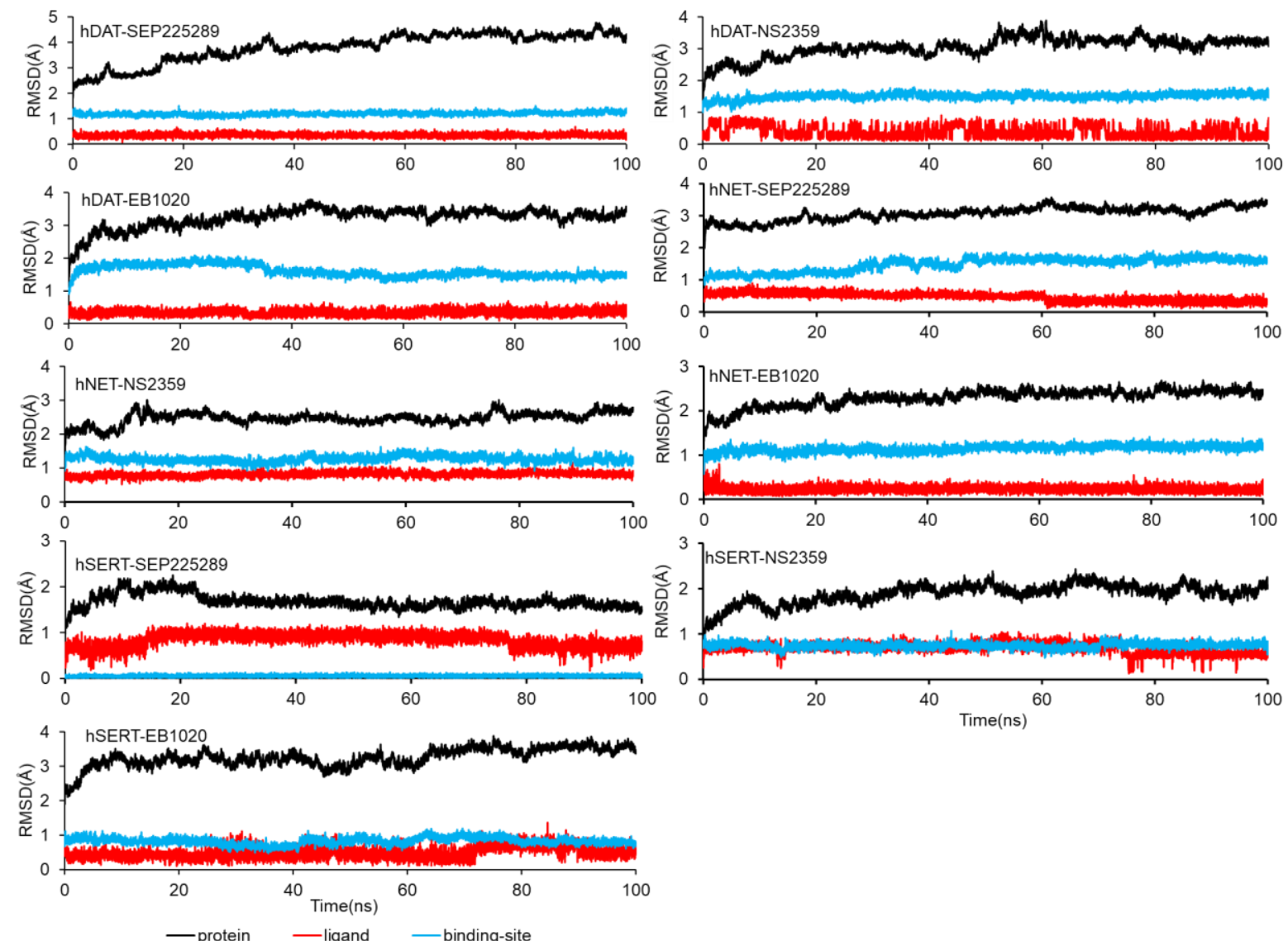

Figure S7. The monitored root-mean-square deviation (RMSD) of protein backbone atoms (black), ligand heavy atoms (red) and binding pocket residues backbone atoms (blue) of the nine complexes during the MD simulation. 

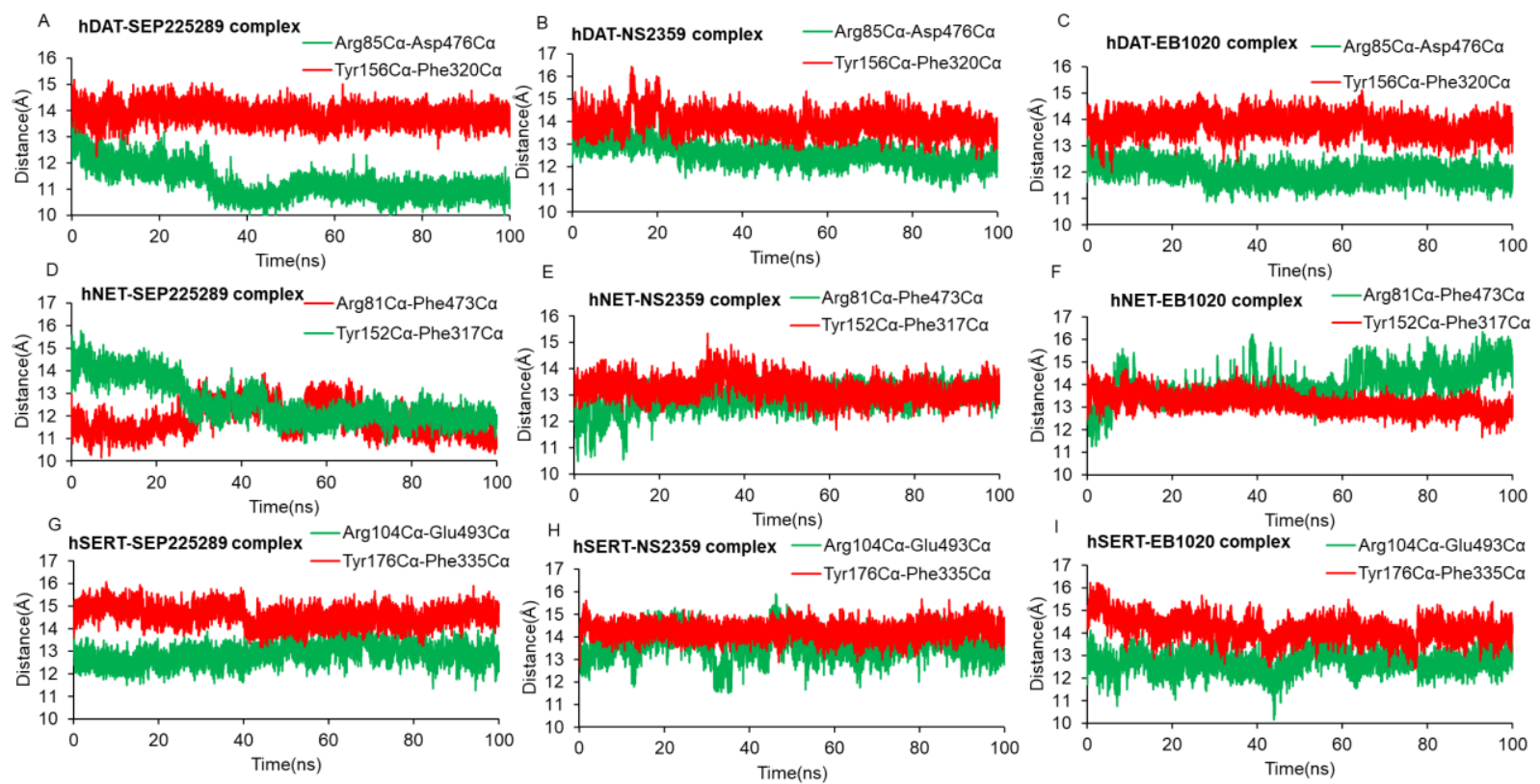

Figure S8. Distances between alpha carbon atoms of gating residues Arg85-Asp476 and Tyr156Phe320 of hDAT(A-C), Arg81-Phe473 and Tyr152-Phe317 of hNET (D-E), Arg104-Glu493 and Tyr176-Phe335 of hSERT (G-I) are $12 \sim 14 \AA, 12-15 \AA$ and 13 16 $\AA$, respectively. 

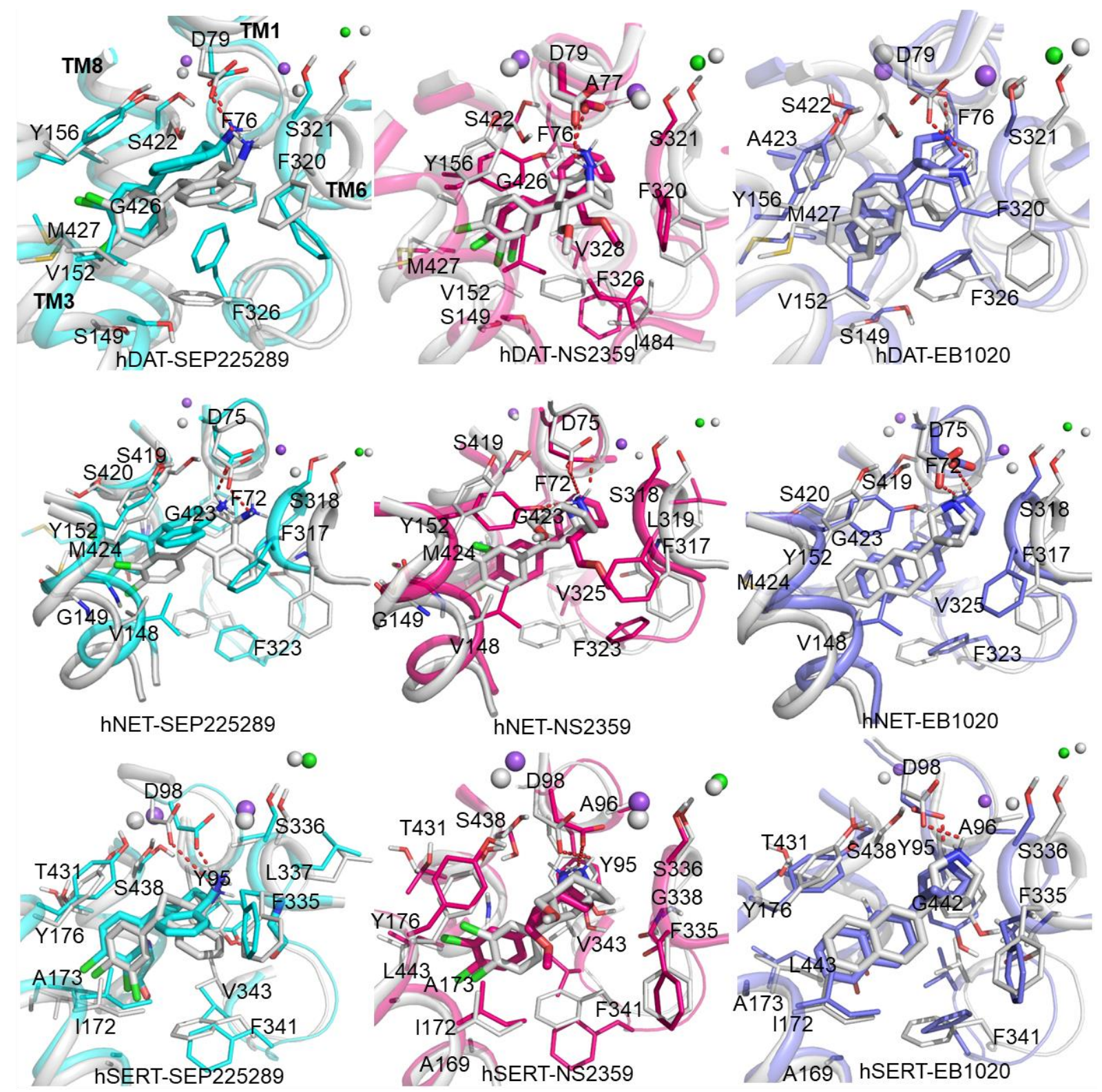

Figure S9. Structural superimposition between the poses of three TRIs in hDAT, hNET and hSERT binding sites obtained by docking (gray) and $\mathrm{MD}$ simulation. The $\mathrm{Na}^{+}$and $\mathrm{Cl}^{-}$are displayed as blue, green balls, respectively. 

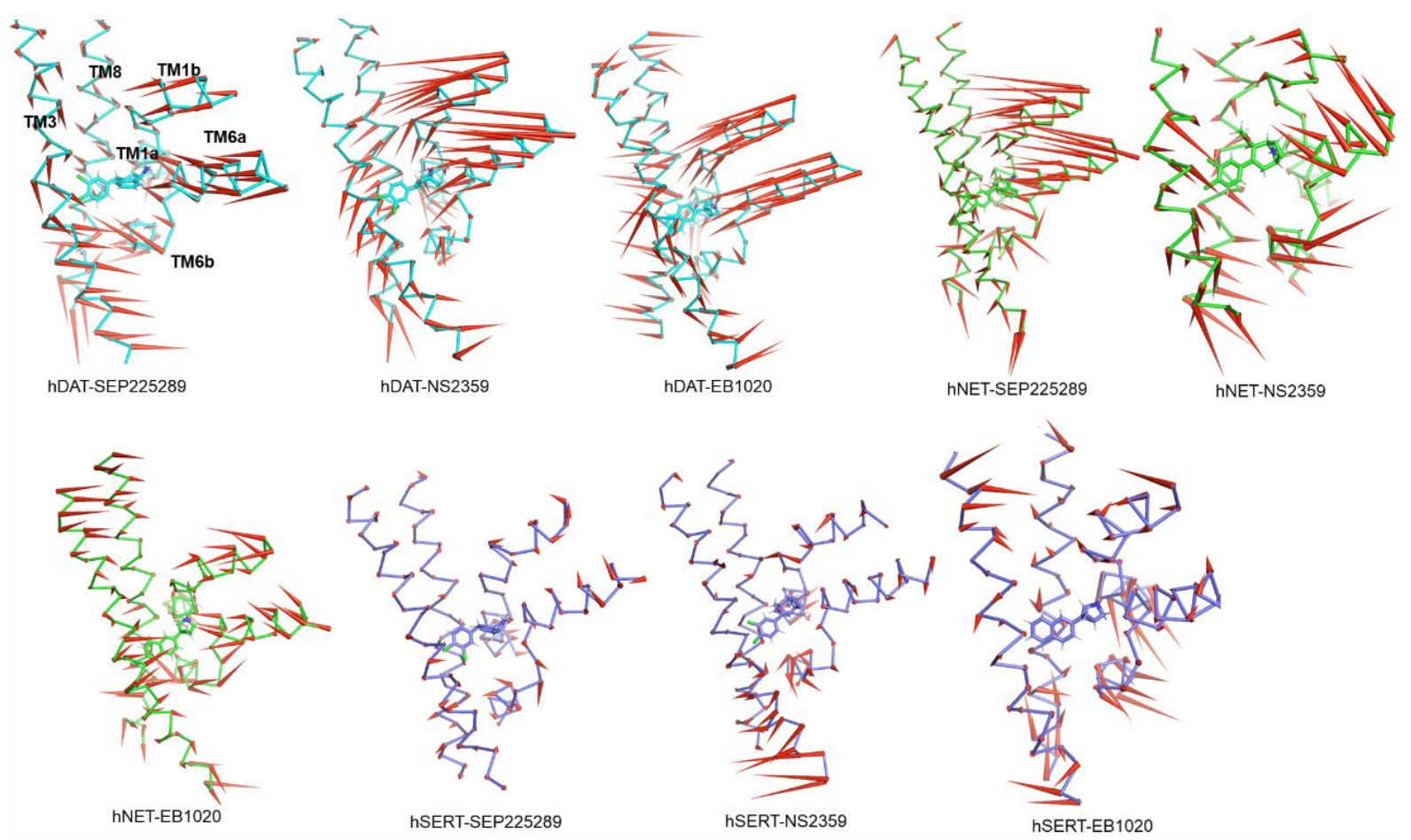

Figure S10. The porcupine plots of three hDAT bound complexes (cyan), hNET bound complexes (green), and hSERT bound complexes (blue), respectively. The red arrows showing the direction of motion of the TM1, TM3, TM6 and TM8 regions along the MD simulation. The PyMOL modevector module ${ }^{5}$ was applied to generate the porcupine plots. 


\section{Reference}

1. Coleman, J. A., Green, E. M., and Gouaux, E. (2016) X-ray structures and mechanism of the human serotonin transporter, Nature 532, 334-339.

2. Subbaiah, M. A. M. (2018) Triple Reuptake Inhibitors as Potential Therapeutics for Depression and Other Disorders: Design Paradigm and Developmental Challenges, J. Med. Chem. 61, 2133-2165.

3. Larkin, M. A., Blackshields, G., Brown, N. P., Chenna, R., McGettigan, P. A., McWilliam, H., Valentin, F., Wallace, I. M., Wilm, A., Lopez, R., et al. (2007) Clustal W and Clustal X version 2.0, Bioinformatics 23, 2947-2948.

4. Robert, X., and Gouet, P. (2014) Deciphering key features in protein structures with the new ENDscript server, Nucleic Acids Res. 42, W320-324.

5. PyMOL Molecular Graphics System, Version 1.3, Schrödinger, LLC. 